\title{
Heterogeneity of Duodenal Neuroendocrine Tumors: An Italian Multi-center Experience
}

\author{
Sara Massironi, MD, $\mathbf{P h D}^{1}$ (D), Davide Campana, $\mathrm{MD}, \mathrm{PhD}^{2}$, Stefano Partelli, $\mathrm{MD}, \mathrm{PhD}^{3}$, Francesco Panzuto, \\ $\mathrm{MD}, \mathrm{PhD}^{4}$, Roberta Elisa Rossi, $\mathrm{MD}^{1}$, Antongiulio Faggiano, $\mathrm{MD}^{5}$, Nicole Brighi, $\mathrm{MD}^{2}$, Massimo Falconi, $\mathrm{MD}^{3}$, \\ Maria Rinzivillo, $\mathrm{MD}^{4}$, Gianfranco Delle Fave, $\mathrm{MD}^{4}$, Anna Maria Colao, $\mathrm{MD}^{5}$, and Dario Conte, $\mathrm{MD}^{1}$ \\ ${ }^{1}$ Gastroenterology and Endoscopy Unit, Fondazione IRCCS Ca' Granda, Ospedale Maggiore Policlinico, Università degli \\ Studi di Milano, Milan, Italy; ${ }^{2}$ Department of Medical and Surgical Sciences, Bologna University St. Orsola-Malpighi \\ Polyclinic Hospital, Bologna, Italy; ${ }^{3}$ Pancreatic Surgery Unit, Pancreas Translational and Clinical Research Center, San \\ Raffaele Scientific Institute, "Vita-Salute" University, Milan, Italy; ${ }^{4}$ Digestive and Liver Diseases Department, University \\ "La Sapienza" of Rome Sant'Andrea Hospital, Rome, Italy; ${ }^{5}$ Division of Endocrinology, Department of Clinical Medicine \\ and Surgery, University "Federico II" of Naples, Naples, Italy
}

\begin{abstract}
Background. The optimal management of duodenal neuroendocrine neoplasms (dNENs) is unclear, and endoscopic resection is increasingly performed instead of surgery.

Methods. This is a retrospective analysis of patients with histologically confirmed diagnosis of dNENs, managed at five Italian tertiary referral Centers in Italy.

Results. From 2000 to 2017, 108 patients (69 males, 39 females, median age 59.5 years) were included in this study. Seventy-one patients had G1, 21 G2, 4 G3 dNENs (12 Ki-67 not available). Fifty-four patients showed metastases at diagnosis, and 20 patients developed metachronous metastases. Thirty patients had a functioning dNEN (14 metastatic). Fifty-seven patients had the dNEN surgically resected, 16 endoscopically, 23 metastatic, received medical therapy + surgery or endoscopy. Seven patients underwent liver-directed therapies, and one patient had PRRT. Median OS was 187 months. During a median follow-up of 76 months, 20 patients died (19 of diseaserelated causes). At Cox's multivariate proportional hazard regression, grading and age were the only variables independently related to OS. Median PFS was 170 months. Grading and staging at the initial diagnosis were
\end{abstract}

(C) Society of Surgical Oncology 2018

First Received: 12 March 2018;

Published Online: 27 July 2018

S. Massironi, MD, PhD

e-mail: sara.massironi@policlinico.mi.it independently related to PFS. No differences in terms of OS and PFS were observed between patients treated surgically or endoscopically.

Conclusions. dNENs prognosis may be highly variable. These tumors can be metastatic in up to $50 \%$ of cases at the time of first diagnosis and can develop metastases thereafter. Functioning neoplasms express high metastatic potential. Nuclear imaging should be performed to exclude distant metastases in all dNENs. Endoscopy and surgery play a primary role in the management of the disease. Further prospective studies are needed.

Duodenal neuroendocrine neoplasms (dNENs) are heterogeneous tumors. They represent up to $3 \%$ of all duodenal tumors and 2-3\% of all gastrointestinal neoplasms. ${ }^{1-3}$ Their incidence has been increasing, reflecting the increased use of upper gastrointestinal endoscopy. ${ }^{4}$ The majority of the cases (50-70\%) are low-grade, well-differentiated tumors, whereas only a minority of the cases are high-grade, poorly differentiated neuroendocrine carcinomas $(<3 \%) .^{5}$ These tumors may be functioning [i.e., gastrinoma (27-58\%), somatostatinoma (23-75\%), secreting serotonin (28\%), calcitonin $(9 \%)$, and rare duodenal gangliocytic paragangliomas] and nonfunctioning. ${ }^{3,6-8}$ The available series often have combined dNENs with smallbowel NENs, even if with a generally more favorable prognosis. ${ }^{4,9}$ Duodenal NENs are usually small in size, limited to the mucosa and submucosa, although metastases in regional lymph nodes are reported in $40-60 \%$ of all cases. ${ }^{5,7}$ Liver metastases occur in less than $10 \%$ of all 
patients. ${ }^{8}$ The majority of dNENs are located in the duodenal bulb (58\%) and descending duodenum (33\%), whilst tumors located in the ampulla of Vater (approximately $20 \%$ ) often are considered as a separate entity due to their clinical behavior more similar to pancreatic neoplasms. ${ }^{10-15}$

Endoscopic resection is increasingly performed instead of surgery. Despite clear differences, in the current guidelines dNEN management is treated along with either gastric or, if functioning, pancreatic NENs. ${ }^{16}$ As a result, their natural history, clinical characteristics, treatment, and prognosis are still poorly understood.

Therefore, the current study first analyzed the natural history of patients with dNENs. The secondary goal was to evaluate the overall (OS) and progression-free survival (PFS), according to the histological features, stage at initial diagnosis, and other possible prognostic parameters.

\section{PATIENTS AND METHODS}

\section{Study Population}

Patients with a histologically confirmed diagnosis of dNENs, as diagnosed and treated from January 2000 to January 2017, at five referral Centers in Italy (Gastroenterology and Endoscopy Unit, Fondazione IRCCS $\mathrm{Ca}$ ' Granda Ospedale Maggiore Policlinico Milano, University of Milan; the Digestive and Liver Diseases Department, University "La Sapienza" of Rome, Sant'Andrea Hospital; the Division of Endocrinology, Department of Clinical Medicine and Surgery, University "Federico II" Naples; the Department of Medical and Surgical Sciences, Bologna University's St. Orsola-Malpighi Hospital; the Pancreatic Surgery Unit, Pancreas Translational and Clinical Research Center, San Raffaele Scientific Institute, University "VitaSalute" Milan) were identified from the database and retrospectively were analyzed.

All consecutive patients who met the following inclusion criteria were enrolled: age $>18$ years; histologically confirmed dNEN; availability of (1) histology (according to the WHO classification), (2) clinical data with a minimum 3-month follow-up after diagnosis, (3) morphologic imaging techniques, such as ultrasound (US), computed tomography (CT), magnetic resonance imaging (MRI), (4) nuclear medicine imaging, (5) endoscopy parameters, (6) biochemical data [Chromogranin A (CgA), gastrin, somatostatin, neuron specific enolase (NSE)]. The exclusion criteria were: histologic finding of MANECs (mixed adenoneuroendocrine carcinomas); age $<18$ years; nonadherence to the written, informed consent to participate in the study; the use of experimental drugs during the 2 months preceding inclusion in this study. The tumors were staged according to the TMN stage scoring system and classified on the basis of their immunohistochemical characteristics according to the WHO 2010 classification, as: dNENs of grade G1 (Ki-67 < 2\%), G2 (Ki-67 3-20\%), and G3 $(\mathrm{Ki}-67>20 \%) .{ }^{17,18}$ The tumors were classified as functioning or nonfunctioning neoplasms. All neoplasms were diagnosed by pathological examination, formalinfixed, and routinely processed. Sections of the tumors were immunostained for CgA, NSE, synaptophysin (SYN), and the Ki-67 proliferative index, using the MIB-I antibody. Functioning tumors were defined as those in which distinct clinical symptoms and excessive levels of specific hormones (i.e., gastrin or somatostatin) were present together with the evidence of predominant or exclusive immunoreactivity to specific hormones. Germline mutations for multiple endocrine type-1 neoplasia (MEN-1) syndrome were tested when clinically suspected, based on the coexistence of dNEN with another major feature of MEN-1 (i.e., primary hyperparathyroidism or pituitary adenoma) or in all cases of gastrinoma.

After the initial diagnosis, regular clinical, biochemical, upper gastro-intestinal (GI) endoscopy, and imaging follow-up examinations were performed for all the cases, at least once a year (range 3-12 months). Conventional radiological imaging, endoscopy, and nuclear medicine imaging (somatostatin receptor scintigraphy or Gallium-68 PET) were performed when clinically indicated. Endoscopic ultrasound was generally performed to assess the degree of wall invasion before resection, in case of $>1-\mathrm{cm}$ lesions. After enrollment, the type of treatment received by each patient was classified as: (1) radical surgery, (2) endoscopic mucosal resection (EMR) or endoscopic submucosal dissection (ESD), (3) follow-up alone, and (4) medical therapy.

Morphological imaging was used to evaluate the objective responses of the disease (i.e., tumor size) according to the criteria released by the Italian Trials in Medical Oncology Group ${ }^{19}$ as: complete or partial response (with a tumor size decrease $>50 \%$ ), stable $(<50 \%$ decrease or $<25 \%$ increase $)$, and progressive (increase $>25 \%$ ).

\section{Data Collection}

All the data were prospectively collected at the Center where the patient had been observed. A single, computerized data sheet was created and for each patient the following data were entered: age, gender, date of diagnosis, age at diagnosis, type of treatment, pathological features, tumor size and localization, number of lesions, time of follow-up, grading according to WHO 2010, staging according to the TNM system, presence/absence of MEN-1 syndrome, functioning/nonfunctioning tumor, OS, PFS. 


\section{Statistical Analysis}

Continuous variables were reported as median (range); categorical variables were reported as count (percentage). All data were checked for distribution normality by the Kolmogoroff-Smirnoff test. The differences between groups were assessed with the Mann-Whitney test and Kruskal-Wallis test as appropriate.

The analysis of the predictive factors of metastatic disease was performed by univariate and multivariate analysis using logistic regression. Predictive factors were expressed as odds ratio (OR) and $95 \%$ confidence interval $(95 \% \mathrm{CI})$. The forward stepwise method was used to build a multivariate model after the inclusion of all variables.

OS was calculated from the date of dNEN diagnosis to the patient's death or the end of data collection. PFS was defined as the time interval between diagnosis and disease progression or the patient's death. The survival curves were estimated using the Kaplan-Meier method, and the logrank test was used for comparing the survival curves between patient groups. The Cox univariate-multivariate regression model was used to analyze the possible association between the variables of interest (TNM staging, Ki67 index, age, gender, primary tumor size and localization, number of lesions, presence of any functioning tumor, and presence of any MEN-1 syndrome) and both the risk of death and the risk of progression. The best multivariate model was identified by using a stepwise forward method (entry criterion: $P<0.05$; removal criterion: $P>0.1$ ). The estimated hazard ratios (HR) - as derived from the Cox models-were reported along with the pertinent 95\% confidence intervals (CIs). A $P$ value $<0.05$, two-sided, was considered statistically significant. The analyses were carried out by software: Graph Pad Prism version 6.00 (GraphPad Software, San Diego, CA) and MedCalc version 17.9.5 (MedCalc Software bvba, Ostend, Belgium).

\section{RESULTS}

In the study period, 108 patients (69 males, 39 females, median age 59.5 [range 18-87] years) were diagnosed and treated at the referral institutions. The patients' characteristics are detailed in Table 1.

Thirty patients had a functioning dNEN (25 gastrinomas, 5 somatostatinomas), of whom 14 were metastatic. Sixteen patients (14.8\%) had MEN-1 syndrome, of whom ten had a functioning dNEN (9 Zollinger-Ellison's syndrome, 1 somatostatinoma) and six had a nonfunctioning dNEN. Seventy-one patients had G1, 21 had G2, and 4 had G3 dNENs according to the WHO 2010 classification. ${ }^{18}$ For 12 patients, the Ki-67 index was not available. dNENs were single in 84 and multiple in 24 patients (median number of lesions 3 , range 2-37). Localization was: bulbar in the majority (44 patients), periampullary in 38 , and located in the second portion of duodenum in 24 (not specified in 2 cases). The median diameter was 12 (range 3-130) $\mathrm{mm}$. Twenty-three of 108 patients were incidentally diagnosed. Overall, 54 patients $(50 \%)$ showed metastases at diagnosis: 12 in the liver, 37 in lymph nodes, and 5 presented both. Twenty patients $(18.5 \%)$ also developed new metachronous metastases, during the follow-up period, of whom 5 de novo and 15 who were already metastatic at diagnosis. During the entire study period, a total of 59 patients $(54.6 \%)$ showed metastatic disease. Fifty-seven patients $(52.7 \%)$ had the dNEN surgically resected, 16 (14.8\%) underwent endoscopic treatment, and 23 (21.3\%), who were metastatic, received a combination of medical therapy (somatostatin analogues [SSA] and chemotherapy $[\mathrm{CT}])$ with either surgery or endoscopy. Among the 57 patients who had undergone surgical therapy, 4 patients showed metastatic disease not detected preoperatively by the combination of morphological and functional imaging. Seven patients underwent liver-directed therapy. Peptide receptor radionuclide therapy (PRRT) was performed only in one patient. The results of the logistic regression for predictive factors of metastatic disease are reported in Table 2.

At univariate analysis grading (OR 5.44; $P=0.003$ ), size (OR 1.03; $P=0.04$ ), functioning status (OR 2.45; $P=0.04)$ and age (OR $0.97 ; P=0.04$ ) were related to the presence of metastases. The location of the primary tumor, number of lesions, gender, and presence of MEN-1 syndrome were not significantly related to the presence of metastases.

At multivariate analysis, age (OR 0.96; $P=0.02$ ), grading (OR 7.7; $P=0.0009$ ), and functioning status (OR $5.8 ; P=0.003)$ were the variables independently related to metastatization.

Over a median 76-month follow-up (range 7-211), 20 patients (18.5\%) died, of whom 19 of disease-related causes. Survival analysis showed a median 187-month OS. Median PFS was 170 months with 21 patients experiencing progression. No differences in terms of both OS and PFS were observed between the patients treated surgically versus endoscopically.

At Cox's multivariate proportional hazard regression, grading (HR 29.6, CI 3.05-288.3, $P=0.0037$ for G3 neoplasia) and age (HR 1.07 CI 1.01-1.13, $P=0.008$ ) were the only variables independently related to OS. As concerns PFS, grading [31.29 (5.8-167.13); $P=0.0001$ for G3 neoplasia] and staging at the initial diagnosis [HR 4.35, CI $1.23-15.40, P=0.02$ for staging IV] were independently related to PFS (Table 3A, B). 
TABLE 1 Baseline characteristics of the patients with duodenal neuroendocrine neoplasms (dNENs)

\begin{tabular}{ll}
\hline Characteristics & N [\%] \\
\hline No. of patients & $108[100]$ \\
Age (yr), median (range) & $59.5(18-87)$ \\
Gender (M/F) & $69 / 39$ \\
Location & \\
Bulb & $44[41]$ \\
Peripapillary & $38[35]$ \\
Descending duodenum & $24[22]$ \\
NA & $2[2]$ \\
Grading & \\
G1 & $71[66]$ \\
G2 & $21[19]$ \\
G3 & $4[4]$ \\
NA & $12[11]$ \\
Diameter (mm), median (range) & $12[3-130]$ \\
Functioning (gastrinoma/somatostatinoma) & $30(25 / 5)[28]$ \\
Nonfunctioning & $78[72]$ \\
Single & $84[78]$ \\
Multiple & $24[22]$ \\
Stage (I, II, III, IV) & $41[38], 13[12], 37[34], 17[16]$ \\
Primary type of treatment & \\
Surgery & $57[53]$ \\
Endoscopy & $16[15]$ \\
Systemic therapy & $23[21]$ \\
Liver-directed therapy & $7[6]$ \\
PRRT & $1[1]$ \\
MEN-1 & $16[15]$ \\
\hline & \\
\hline
\end{tabular}

TABLE 2 Results of the logistic regression for predictive factors (covariates) at diagnosis of the development of metastatic disease at univariate and multivariate analyses

\begin{tabular}{lllll}
\hline Covariate & $\begin{array}{l}\text { Univariate } \\
P \text { value }\end{array}$ & OR $(95 \% \mathrm{CI})$ & $\begin{array}{l}\text { Multivariate } \\
P \text { value }\end{array}$ & OR (95\% CI) \\
\hline Age & $0.04^{*}$ & $0.97(0.94-0.99)$ & $0.02^{*}$ & $0.96(0.92-0.99)$ \\
Grading & $0.003^{*}$ & $5.44(1.78-16.56)$ & $0.0009^{*}$ & $7.72(2.31-25.83)$ \\
Size & $0.04^{*}$ & $1.03(1.00-1.07)$ & 0.36 & $1.01(0.98-1.05)$ \\
Functioning status & $0.04^{*}$ & $2.45(1.00-6.03)$ & $0.0036^{*}$ & $5.87(1.78-9.38)$ \\
Site primary & 0.09 & $1.55(0.92-2.62)$ & - & - \\
Number of the lesions & 0.39 & $0.94(0.8-1.08)$ & - & - \\
Gender & 0.49 & $1.31(0.59-2.91)$ & - & - \\
MEN-1 & 0.88 & $1.08(0.37-3.14)$ & - & - \\
\hline A $P$ value & 0.05 was & & \\
\hline
\end{tabular}

A $P$ value $<0.05$ was considered as statistically significant $(*)$

\section{DISCUSSION}

The present multicentric study confirmed that dNENs are heterogeneous tumors characterized by highly variable prognosis, according to the previously available data. dNENs often have been considered similar to gastric NENs, because they are usually small, well-differentiated, and indolent. However, they can be metastatic in up to $54.6 \%$ of cases, as observed in the present series, either at diagnosis or thereafter.
This observation should be taken into account, also on consideration that the median diameter of the lesions in this series was $12 \mathrm{~mm}$, which is very small. In view of their potential aggressive behavior, and given that dNENs tend to spread to the submucosal layer even during the early stages of the disease, surgical resection has been suggested as the preferred treatment modality over endoscopic treatment. Moreover, the duodenal wall is thinner than the gastric wall, and this is possibly a reason to consider first towards surgery versus endoscopic approach, due to the 
TABLE 3 Covariates in relation to overall survival (A) and progression-free survival (B)

\begin{tabular}{llcll}
\hline Covariate & $\begin{array}{l}\text { Univariate } \\
P \text { value }\end{array}$ & HR $(95 \% \mathrm{CI})$ & $\begin{array}{l}\text { Multivariate } \\
P \text { value }\end{array}$ & HR $(95 \% \mathrm{CI})$ \\
\hline (A) Overall survival & & & & \\
Grading (G2) & 0.32 & $1.86(0.54-6.30)$ & - & - \\
Grading (G3) & $0.0001^{*}$ & $44.26(10.81-181.38)$ & $0.0037^{*}$ & $29.6(3.05-288.3)$ \\
Age & $0.0002^{*}$ & $1.06(1.02-1.10)$ & $0.008^{*}$ & $1.07(1.01-1.13)$ \\
No. of lesions & $0.041^{*}$ & $0.35(0.06-1.94)$ & 0.51 & $0.69(0.23-2.05)$ \\
Size & 0.46 & $1.0(0.99-1.02)$ & - & - \\
Stage & 0.73 & $1.2(0.47-2.86)$ & - & - \\
Site primary & 0.47 & $1.9(0.61-6.50)$ & - & - \\
Functioning status & 0.99 & $1.0(0.37-2.069)$ & - & - \\
Gender & 0.47 & $0.68(0.24-1.91)$ & - & - \\
MEN-1 & 0.15 & $0.29(0.03-1.19)$ & - & $1.64(0.43-6.28)$ \\
(B) Progression-free & survival & & & $31.29(5.8-167.13)$ \\
Grading (G2) & $0.04^{*}$ & $3.07(1.03-9.16)$ & 0.47 & - \\
Grading (G3) & $0.0001^{*}$ & $39.29(9.70-159.08)$ & $0.0001^{*}$ & - \\
Age & 0.24 & $1.01(0.98-1.05)$ & - & - \\
No. of lesions & 0.68 & $0.92(0.64-1.33)$ & - & - \\
Size & 0.63 & $0.99(0.97-1.01)$ & - & $-35(1.23-15.40)$ \\
Stage (stage IV) & $0.0007^{*}$ & $6.40(2.24-18.29)$ & $0.02^{*}$ & - \\
Site primary & 0.87 & $0.91(0.35-2.35)$ & - & - \\
\hline
\end{tabular}

risk of perforation. ${ }^{20-28}$ Surgery often is suggested as the treatment of choice because of the risk of lymph node metastases with these tumors and the poor detection rate of conventional imaging for micrometastases. ${ }^{28}$ Conversely, the endoscopic resection of dNENs is increasingly performed instead of surgery and it has proved to be safe and effective only for lesions of $\leq 10 \mathrm{~mm}$ size, confined to the submucosal layer, with no lymph node involvement or distant metastasis. ${ }^{29-35}$ In a recent series of $38 \mathrm{dNEN}$ patients diagnosed over a 5-year period, no recurrence was observed over a mean 17-month follow-up period, and endoscopic submucosal dissection (ESD) achieved a higher rate of radical excision than endoscopic mucosal resection (EMR). ${ }^{29}$ Therefore, both surgery and endoscopy play a primary role in the management of the disease for curative purposes, even if the efficacy of endoscopic treatment in comparison to surgical therapy has not been systematically evaluated. In our multicenter study, we did not observe any significant difference in terms of both OS and PFS between the patients surgically versus endoscopically treated. The majority of patients endoscopically treated had been incidentally diagnosed and exhibited smaller lesions and lower stages. Obviously, this is a retrospective study, so this data cannot be considered as a base of evidence-based equivalence between the two treatments. However, surgery should be performed for tumors $>1 \mathrm{~cm}$ and/or involving the muscolaris propria or in those with positive margins after endoscopic resection. ${ }^{16}$
Because of the high metastatic potential observed in the present study, in case of dNENs, an initial complete staging should be performed before surgery or endoscopic treatment, including nuclear medicine imaging, to exclude distant metastases. Moreover, because of the heterogeneity of these neoplasms and the risk of local and distant metastases (also observed during the study), long-term follow-up is necessary after their initial endoscopic or surgical resection.

The present series has showed that functioning neoplasms express higher metastatic potential and appear to be more aggressive compared with nonfunctioning forms, differently from the pancreatic NENs. ${ }^{36}$ In our series, we have defined the tumors as "functioning" only in the presence of distinct clinical symptoms and excessive levels of specific hormones (i.e., gastrin or somatostatin) together with the evidence of predominant or exclusive immunoreactivity to specific hormones. According to these criteria, we observed that 14 of 30 functioning dNENs (25 gastrinomas, 5 somatostatinomas) were metastatic. This finding is in line with the recent study by Vanoli et al. ${ }^{6}$ who identified five types of NENs with distinct clinicopathological profiles among more than 200 neoplasms arising in the duodenal tract. They showed that the ampullary-type, somatostatin-producing tumors and gastrinomas presented with high rates of local infiltration (especially lympho-invasion and deep duodenal wall/pancreatic tissue invasion) 
and lymph node metastases, whereas the nonfunctioning forms had significantly lower and more size-dependent local invasive potential.

Besides the functioning status, age and grading were the variables independently related to metastatization. Furthermore, grading and age were the only variables independently related to OS, and grading and staging at initial diagnosis were independently related to PFS. Based on these observations, the identification of subgroups of patients with a potentially more aggressive disease (e.g., elderly patients with specific histological characteristics or presence of a clinical syndrome) who deserve a more aggressive therapeutic approach (i.e., surgery) should be the ultimate goal.

Finally, in this series we have observed 16 patients (14.8\%) with MEN-1 syndrome. This is an interesting finding as the association with MEN-1 has been historically better defined for pancreatic NENs. ${ }^{37}$ Thus, one should keep in mind that also in cases of dNENs, either functioning or nonfunctioning, MEN-1 syndrome should be considered. $^{38}$ This is in line with previous observations in which MEN-1 has reportedly occurred in $20-30 \%$ of all patients with dNENs with Zollinger-Ellison's syndrome $(\mathrm{ZES})^{37},{ }^{39},{ }^{40}$ : Vanoli et al. found 7 MEN-1 cases among 20 gastrinoma patients in their retrospective study (i.e., $35 \%$ of gastrinomas but only approximately $4 \%$ of the entire cohort). ${ }^{6}$ In our series, the patients with MEN-1 syndrome presented gastrinomas and also nonfunctioning dNENs (a somatostatinoma was observed in one case).

A possible limitation of this study is a degree of unavoidable variability among the monitoring or treating methods because of its multi-institutional retrospective nature. Nevertheless, from this multicenter study, we can derive worthwhile information about this kind of NENs. In fact, the present study has depicted dNENs as heterogeneous tumors that can exhibit an aggressive behavior with distant metastases, despite their small size, more frequently than previously described. Therefore, careful disease staging, including nuclear medicine testing, and a radical treatment approach should be considered. Follow-up should be extended to a lifelong horizon.

Further prospective studies are needed to better define standardized guidelines dedicated to dNENs, including optimal patient treatment and management and effective follow-up intervals.

ACKNOWLEDGMENT We thank It.a.net for the support.

CONFLICT OF INTEREST None.

\section{REFERENCES}

1. Attanoos R, Williams GT. Epithelial and neuroendocrine tumors of the duodenum. Semin Diagn Pathol. 1991;8:149-62.

2. O'Toole D, Delle Fave GD, Jensen RT. Gastric and duodenal neuroendocrine tumours. Best Pract Res Clin Gastroenterol. 2012;26:719-35.

3. Jensen RT, Rindi G, Arnold R, et al.; Frascati Consensus Conference; European Neuroendocrine Tumor Society. Welldifferentiated duodenal tumor/carcinoma (excluding gastrinomas). Neuroendocrinology. 2006;84:165-72.

4. Fitzgerald TL, Dennis SO, Kachare SD, et al. Increasing incidence of duodenal neuroendocrine tumors: incidental discovery of indolent disease? Surgery. 2015;158(2):466-71. https://doi.or g/10.1016/j.surg.2015.03.042. (Epub 2015 May 23).

5. Lipiński M, Rydzewska G, Foltyn W, et al. Gastroduodenal neuroendocrine neoplasms, including gastrinoma-management guidelines (recommended by the polish network of neuroendocrine tumours). Endokrynol Pol. 2017;68(2):138-53.

6. Vanoli A, La Rosa S, Klersy C, et al. Four neuroendocrine tumor types and neuroendocrine carcinoma of the duodenum: analysis of 203 cases. Neuroendocrinology. 2017;104:112-25.

7. Kloppel G, Perren A, Heitz PU. The gastroenteropancreatic neuroendocrine cell system and its tumors: the WHO classification. Ann N Y Acad Sci. 2004;1014:13-27.

8. Hoffmann KM, Furukawa M, Jensen RT. Duodenal neuroendocrine tumors: classification, functional syndromes, diagnosis and medical treatment. Best Pract Res Clin Gastroenterol. 2005;19:675-97.

9. Modlin IM, Champaneria MC, Chan AK, et al. A three-decade analysis of 3911 small intestinal neuroendocrine tumors: the rapid pace of no progress. Am J Gastroenterol. 2007;102:1464-73.

10. Hatzitheoklitos E, Buchler MW, Friess H, et al. Carcinoid of the ampulla of Vater. Clinical characteristics and morphologic features. Cancer. 1994;73:1580-8.

11. Walton GF, Gibbs ER, Spencer GO, et al. Carcinoid tumors of the ampulla of Vater. Am Surg. 1997;63:302-4.

12. Bornstein-Quevedo L, Gamboa-Dominguez A. Carcinoid tumors of the duodenum and ampulla of vater: a clinicomorphologic, immunohistochemical, and cell kinetic comparison. Hum Pathol. 2001;32:1252-6.

13. Witzigmann H, Loracher C, Geissler F, et al. Neuroendocrine tumours of the duodenum. Clinical aspects, pathomorphology and therapy. Langenbecks Arch Surg. 2002;386:525-33.

14. Clements WM, Martin SP, Stemmerman G, et al. Ampullary carcinoid tumors: rationale for an aggressive surgical approach. $J$ Gastrointest Surg. 2003;7:773-6.

15. Hartel M, Wente MN, Sido B, et al. Carcinoid of the ampulla of Vater. J Gastroenterol Hepatol. 2005;20:676-81.

16. Delle Fave G, O'Toole D, Sundin A, et al. Vienna consensus conference participants: ENETS consensus guidelines update for gastroduodenal neuroendocrine neoplasms. Neuroendocrinology. 2016;103:119-24.

17. Rindi G, Klöppel G, Alhman H, et al.; all other Frascati Consensus Conference participants; European Neuroendocrine Tumor Society (ENETS); European Neuroendocrine Tumor Society (ENETS). TNM staging of foregut (neuro)endocrine tumors: a consensus proposal including a grading system. Virchows Arch. 2006;449:395-401.

18. Rindi G, Arnold R, Bosman FT, et al. Nomenclature and classification of neuroendocrine neoplasms of the digestive system. In: Bosman FT, Carneiro F, Hruban RH, Theise ND, editors. WHO classification of tumors of the digestive system. Lyon: International Agency for Research on Cancer (IRAC); 2010:13-4. 
19. Bajetta E, Zilembo N, Di Bartolomeo M, et al. Treatment of metastatic carcinoids and other neuroendocrine tumors with recombinant interferon-alpha-2a. A study by the Italian Trials in Medical Oncology Group. Cancer. 1993;72:3099-105.

20. Tsujimoto $H$, Ichikura $T$, Nagao $S$, et al. Minimally invasive surgery for resection of duodenal carcinoid tumors: endoscopic full-thickness resection under laparoscopic observation. Surg Endosc. 2010;24:471-5.

21. Dogeas E, Cameron JL, Wolfgang CL, et al. Duodenal and ampullary carcinoid tumors: size predicts necessity for lymphadenectomy. J Gastrointest Surg. 2017;21:1262-9.

22. Iwasaki T, Nara S, Kishi Y, et al. Surgical treatment of neuroendocrine tumors in the second portion of the duodenum: a single center experience and systematic review of the literature. Langenbecks Arch Surg. 2016. (Epub ahead of print).

23. Hatta W, Koike T, Iijima K, et al. The risk factors for metastasis in non-ampullary duodenal neuroendocrine tumors measuring $20 \mathrm{~mm}$ or less in diameter. Digestion. 2017;95:201-9.

24. Carter JT, Grenert JP, Rubenstein L, et al. Neuroendocrine tumors of the ampulla of Vater: biological behavior and surgical management. Arch Surg. 2009;144:527-31.

25. Makhlouf HR, Burke AP, Sobin LH. Carcinoid tumors of the ampulla of Vater: a comparison with duodenal carcinoid tumors. Cancer. 1999; 85:1241-1219.

26. Burke AP, Sobin LH, Federspiel BH, et al. Carcinoid tumors of the duodenum. A clinicopathologic study of 99 cases. Arch Pathol Lab Med. 1990;114:700-4.

27. Gincul R, Ponchon T, Napoleon B, et al. Endoscopic treatment of sporadic small duodenal and ampullary neuroendocrine tumors. Endoscopy. 2016;48:979-86. (Epub 2016 Aug 5).

28. Pedicone R, Adham M, Hervieu V, et al. Long-term survival after pancreaticoduodenectomy for endocrine tumors of the ampulla of Vater and minor papilla. Pancreas. 2009;38:638-43. https://doi. org/10.1097/mpa.0b013e3181a9d41f.

29. Kim GH, Kim JI, Jeon SW, et al; Korean College of Helicobacter and Upper Gastrointestinal Research. Endoscopic resection for duodenal carcinoid tumors: a multicenter, retrospective study. $J$ Gastroenterol Hepatol. 2014;29:318-24.
30. Fukasawa H, Tounou S, Nabetani M, et al. Endoscopic resection of ampullary neuroendocrine tumor. Intern Med. 2017;56:499-503.

31. Yokoyama S, Takifuji K, Tani M, et al. Endoscopic resection of duodenal bulb neuroendocrine tumor larger than $10 \mathrm{~mm}$ in diameter. BMC Gastroenterol. 2011;11:67.

32. Gilani N, Ramirez FC. Endoscopic resection of an ampullary carcinoid presenting with upper gastrointestinal bleeding: a case report and review of the literature. World $J$ Gastroenterol. 2007; 13:1268-70.

33. Fukatsu H, Kawamoto H, Fujii M, et al. Periampullary carcinoid tumor. Endoscopy. 2007; Suppl 1:E49-50.

34. Yi H, Wu C, Mou Y, et al. Successful en bloc resection of papillary neuroendocrine tumors by duodenoscope using endoscopic submucosal dissection method. Clin Res Hepatol Gastroenterol. 2012;36:e100-3.

35. Odabasi M, Yildiz KM, Cengiz E, et al. Treatment of ampullary neuroendocrine tumor by endoscopic snare papillectomy. Am J Case Rep. 2013;14:439-43.

36. Falconi M, Eriksson B, Kaltsas G, et al; Vienna Consensus Conference participants. ENETS Consensus Guidelines update for the management of patients with functional pancreatic neuroendocrine tumors and non-functional pancreatic neuroendocrine tumors. Neuroendocrinology. 2016;103(2):153-71.

37. Gibril F, Schumann M, Pace A, et al. Multiple endocrine neoplasia type 1 and Zollinger-Ellison syndrome: a prospective study of 107 cases and comparison with 1009 patients from the literature. Medicine. 2004;83:43-83.

38. Plöckinger U. Diagnosis and treatment of gastrinomas in multiple endocrine neoplasia type 1 (MEN-1). Cancers. 2012;4:39-54.

39. Jensen RT. Management of the Zollinger-Ellison syndrome in patients with multiple endocrine neoplasia type 1. J Intern Med. 1998;243:477-88.

40. Norton JA, Fraker DL, Alexander HR, et al. Surgery to cure the Zollinger-Ellison syndrome. N Engl J Med. 1999;341:635-44. 